\title{
Subjective contours and the Poggendorff illusion
}

\author{
GLENN E. MEYER and CAROL GARGES \\ Lewis and Clark College, Portland, Oregon 97219
}

\begin{abstract}
Poggendorff illusions were generated by real edges, subjective contours, and various control patterns. Using both magnitude estimation and reproduction measures of illusion strength, it was found that subjective contours produced a reliable Poggendorff illusion. This clarifies previous reports which could not demonstrate a subjective contour-based illusion.
\end{abstract}

Recently, there has been some discussion as to whether the illusory edges produced by a subjective contour can induce the perceptual phenomena apparent with real lines or edges. Tilt aftereffects and increment threshold changes have been reported with subjective contours (Coren \& Theodor, 1977; Smith \& Over, 1975). Gregory (1972) has also reported a version of the Poggendorff illusion in which the apparent misalignment of the interrupted oblique line is due to subjective rather than real edges.

However, Day, Dickinson, and Jory (1977) have questioned Gregory's claim due to its informal nature and the tendency of oblique lines to be misaligned even in the absence of any type of edge, contour, or line. Day et al. did not report a subjective contour version of the Poggendorff illusion of a greater magnitude than that seen with several control configurations which did not evoke powerful illusory contours. In spite of this negative finding, Day et al.'s (1977) study was not conclusive on this issue. Their data were collected by having subjects adjust the position of the misaligned oblique lines. Day et al. felt that the intersection of these lines with the semicircular elements inducing the subjective contours in their displays may have negated the Poggendorff illusion.

The present study first examined the reality of a subjective contour version of the Poggendorff illusion by using magnitude estimation of illusory strength, using configurations which avoided the possible destructive interactions involved in moving the oblique line segments. Since such ranking procedures are not particularly powerful techniques, the results were replicated using a method of reproduction which Coren and Girgus (1972) have shown to be a reliable indicator of an illusion's strength.

Six configurations were tested in both experiments (see Figure 1). They included the standard illusion with inducing patterns of real edges, a subjective contour version with illusory edges produced by dark

Experiment 1 was presented at the 1977 annual meeting of the Association for Research in Vision and Ophthalmology in Sarasota, Florida. semicircular elements, and a display containing no illusion-producing elements. There were three other versions of the illusion for further comparison and control purposes. The first was an outline version of the subjective contour display. It contained similar features and cues as to perspective and angles in the subjective contour display, but produced much weaker illusory contours. The next type consisted of solid "dots" in the same location as the elements of the subjective contour display, to provide a control condition with reduced contour but equal brightness contrast although not producing an edge. The final condition was an outline version of the solid dot pattern or an "empty" dot configuration. We hoped that the subjective contour illusions would prove more powerful than these various control conditions.

\section{EXPERIMENT 1}

\section{Method}

Subjects. Thirty-four men and women from an introductory psychology class were used. All had normal or corrected vision. They were naive to the nature of the illusion and the purpose of the experiment.

Stimuli. An $81 / 2 \times 11$ in. inspection sheet with the Poggendorff illusion was composed and then duplicated. On each sheet were the six versions of the illusion, with inducing contexts constructed from solid dots, subjective contours, real contours, empty dots, empty contours (outlines of the edges that produce subjective contours), and no contour (Figures 1A, 1B, 1C, 1D, 1E, and 1F, respectively). Line width was $1 \mathrm{~mm}$. The parameters of the real illusion were chosen from various sources (Julesz, 1971; Robinson, 1972 ) to produce a strong effect. The dimensions of the illusion were as follows: The rectangle was $2 \times 7 \mathrm{~cm}$, and the intercepting lines were $2 \mathrm{~cm}$ long, touching the rectangle at $2 \mathrm{~cm}$ from its ends, making an angle of $40^{\circ}$ with the vertical sides of the rectangle; the diameter of the large dots, which were also used to construct the subjective contours, was $7 \mathrm{~mm}$; the small dots were $5 \mathrm{~mm}$ in diameter.

The subject viewed the sheets at a distance of 50 to $65 \mathrm{~cm}$. Fluorescent room lighting was measured at $28 \mathrm{fL}$ on the white sheets. Contrast ratio of the black components to the white background was .51 .

Procedure. The subjects were seated, and an example of the Poggendorff illusion (see Figure 1C) was shown to them. The apparent displacement of the target line was explained. A display containing the six illusion versions (a duplicate of Figure 1) then was presented. The subjects were told to rate the size of the apparent displacement of the diagonal line for each illusion on a 


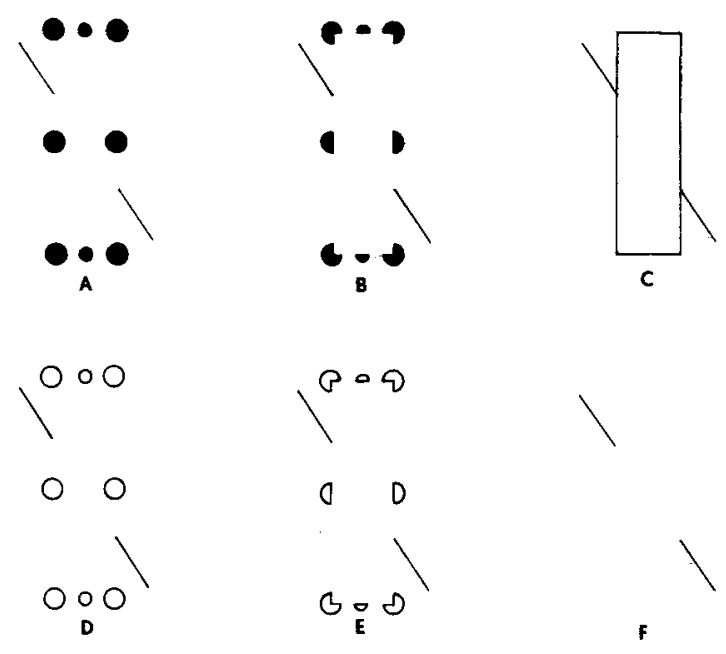

Figure 1. Poggendorff illusion variants. Top row, left to right: solid dots (A), subjective contours (B), real contours (C), empty dots (D), empty contours (E), and no contour (F).

5 to 0 basis, with ties allowed. The responses were written. There was no time limit, but the subjects were quick in their responding. No subject had any difficulty with this task. Pilot work, using three configurations (Figures 1B, 1C, and 1F) had previously produced reliable results.

\section{Results}

The data for the six inducing configurations are presented in Figure 2. A within-subject ANOVA revealed a significant effect of contour type $[F(5,165)$ $=9.15, \mathrm{p}<.01]$. As can be seen, the real contours were most effective in inducing the illusions, followed by the subjective contours (Figure 1B). For all the illusions, however, the various configurations (Figures 1A-1E) produced illusions as compared to the no-contour (Figure 1F) condition.

\section{Discussion}

It is apparent that naive subjects consistently rate the subjective contour version of the Poggendorff illusion as greater in magnitude than the control conditions which contained similar lines and angles ("empty" contours) and contrast (solid dots) but produced weak or no illusory edges. However, magnitude estimation with all illusions present may contain some hidden artifactual components due to the necessity of explaining the Poggendorff's displacement effect to the subject. Also, Coren and Girgus (1972) have compared five methods of measuring illusion magnitude, and reported magnitude estimation was not one of the most satisfactory techniques, although it does produce interpretable data. Therefore, the experiment was replicated using reproduction, a method that Coren and Girgus' study reports as quite reliable, with each illusion presented separately to avoid any unknown interactions that may have existed in the six-figure display and without informing subjects of the nature of the illusion by just asking for a judgment of whether the target lines met.

\section{EXPERIMENT 2}

\section{Method}

Subjects. Sixteen men and women from an undergraduate perception class were used. All had normal or corrected vision. At time of testing, they were naive as to the nature of the illusion and the purpose of the experiment.

Stimuli. Each of the six versions of the Poggendorff illusion presented in Figure 1 was drawn on a separate master copy and then duplicated. The individual illusions were of the same dimensions and contrast as in Figure 1 and were presented under the same lighting conditions. Also prepared was a $2-\mathrm{cm}$ standard line centered on a similar sheet.

Procedure. The subject was seated, and each version of the Poggendorff was presented to the subject along with a standard line oriented vertically. The subject was instructed to mark off carefully the size of misalignment of the oblique lines, if any, on the standard line, using the bottom of the line as a starting point. Order of presentation was randomized.

\section{Results}

The reproduction data are presented in Figure 3 and show the same pattern as found in Experiment 1. Again, the real illusion was of the greatest magnitude, followed by the subjective contour version. The "empty" contour, "empty" dots, and solid dots were grouped together at a lower level with the "nocontour" pair of isolated oblique lines showing the least displacement. This is reflected in a significant within-group $\operatorname{ANOVA}[\mathrm{F}(5,75)=5.07, \mathrm{p}<.01]$. A posteriori Tukey tests between all possible pairs found that means of the real contour, subjective contour, and no-contour Poggendorffs differed from each other and the other control conditions $(p<.01)$. The solid dot, empty dot, and empty contour grouping showed no significant differences $(p>.05)$ among themselves.

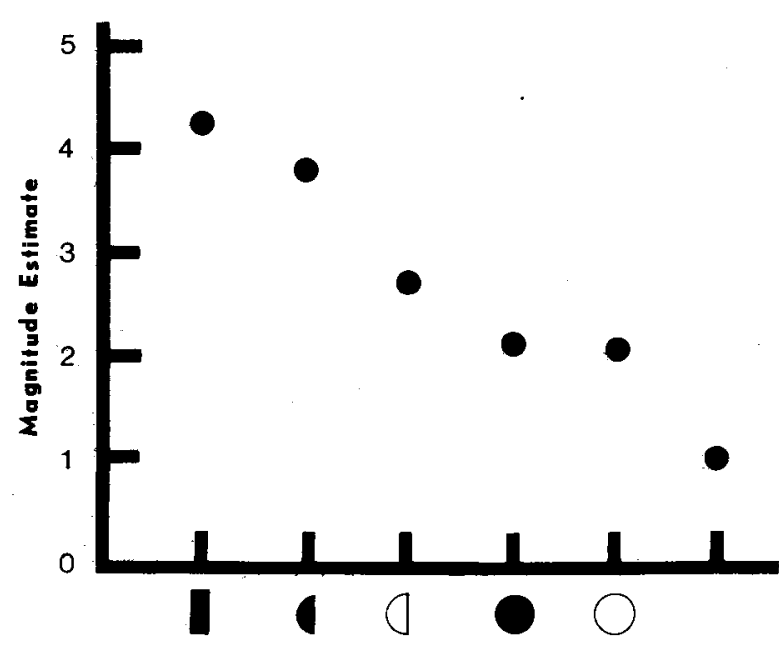

Figure 2. Magnitude estimates for six Poggendorff variants. Abscissa represents real contours, subjective contours, "empty" contours, solid dots, empty dots, and no-contour versions. 


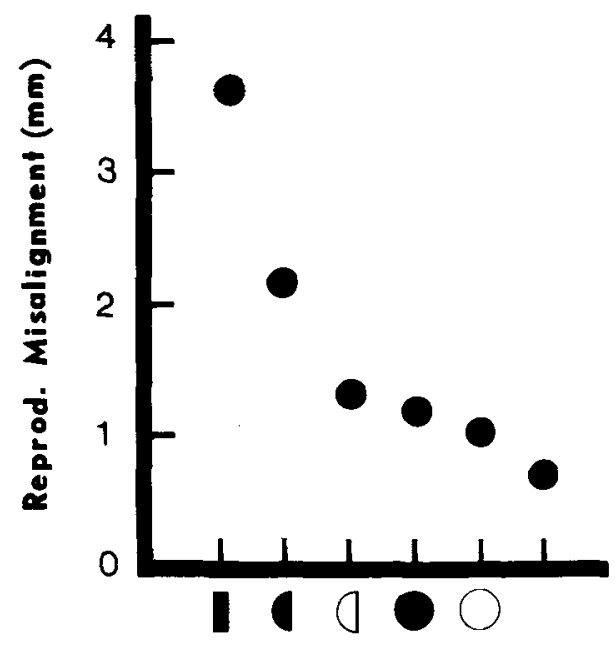

Figure 3. Reproduction data for six Poggendorff variants.

\section{Discussion}

It is apparent from Experiments 1 and 2 that a subjective contour can induce the Poggendorff illusion. Since the reproduction data replicated the qualitative findings of Experiment 1 and produced effects greater than those of the control conditions, we can assume that Day et al.'s failure to find an illusion is not conclusive. If the intersection confound of the Day et al. study is eliminated, as in this study's displays, the subjective contour illusion is clearly present. Day et al. did not rule out this possibility.

It has been postulated that subjective contours might be due to implicit depth cues in the two-dimensional stimulus array (Coren, 1972) or involve some form of neural interactions, such as lateral inhibition or the networks of orientation-specific cortical neurons (Coren \& Theodor, 1977; Smith \& Over, 1975). Day and Jory (1978) suggest that dissimilation of brightness (an enhancement of brightness at the end of a line) and assimilation of brightness (the lessening of brightness in between lines) may also contribute to subjective contour formation, but they do not postulate a neurophysiological correlate (but see Frisby \& Clatworthy, 1975). Similar analyses of depth and/or neural interactions are commonly evoked as explanations of various illusions (Gregory, 1970; Julesz, 1971; Robinson, 1972). The present experiment does not differentiate clearly between these possibilities. However, the "empty contour" (Figure 1E) condition contained the same information as relates to lines, intersections, and angles, as did the subjective contour display, but did not generate a strong illusion as compared to the dot controls or generate a strong illusory rectangle. The lack of strong subjective contour in this figure is in accord with the analyses of Day and Jory (1978) and Frisby and Clatworthy (1975). The lines of the empty elements produce less brightness contrast and/or dissimilation of brightness.
Perhaps, then, the second class of neuronal explanations mentioned above might fit the current set of findings. Thus, if neuronal processes are involved in generating the subjective edge, one might expect this edge to be effective in the possible neuronal interactions that might yield the Poggendorff. A weak edge produces a weak line misalignment.

One final point, Coren (1970), as well as this study, produced the Poggendorff with dot patterns. He felt that his data minimized the role, if any, of retinal inhibition in these illusions as the contours necessary (i.e., lines) for the operation of the inhibitory mechanisms were removed. This logic is not necessarily convincing for cortical mechanisms. Inhibitory interactions occur there, and we do not fully understand the effects of dot patterns on orientation-specific processes, but it is possible that they activate them through receptive field summation rather than totally bypass them. Dot patterns do produce weak tilt aftereffects (Smith \& Over, 1975). Therefore, production of a dot-pattern illusion does not mean the elimination of all inhibitory effects in the visual system and the generation of a purely cognitive illusion. Our present knowledge does not support a strong statement, as the residual illusion in the dot cases could just be residual inhibition. Thus, the successful production of the illusion in this study by dot patterns cannot, by itself, demonstrate an illusion free of inhibitory influences.

\section{REFERENCES}

Coren, S. Lateral inhibition and geometric illusions. Quarterly Journal of Experimental Psychology, 1970, 22, 274-278.

Conen, S. Subjective contours and apparent depth. Psychological Review, 1972, 79, 359-367.

Coren, S., \& Girgus, J. S. A comparison of five methods of illusion measurement. Behavior Research Methods and Instrumentation, 1972, 4, 240-244.

Coren, S., \& Theodor, L. H. Neural interactions and subjective contours. Perception, 1977, 6, 107-111.

Day, R. H., Dickinson, R. G., \& JoRy, M. K. The Poggendorff illusion with subjective contours. Quarterly Journal of Experimental Psychology, 1977, 29, 219-226.

DAY, R. H., \& JoRY, M. K. Subjective contours, visual acuity, and line contrast. In J. C. Armington, J. Krauskopf, \& B. R. Wooten (Eds.), Visual psychophysics and physiology. New York: Academic Press, 1978.

Frisby, J. P., \& Clatworthy, J. L. Illusory contours: Curious cases of simultaneous brightness contrast? Perception, 1975, 4, 349-357.

Gregory, R. L. The intelligent eye. New York: McGraw-Hill, 1970.

Gregory, R. L. Cognitive contours. Nature, 1972, 238, $51-52$.

Julesz, B. Foundations of cyclopean perception. Chicago: University of Chicago Press, 1971.

RoBinson, J. O. The psychology of visual illusions. London Hutchinson, 1972.

Smith, A., \& Over, R. Tilt aftereffects and subjective contours Nature, 1975, 257, 581-582.

(Received for publication January 22, 1979; revision accepted August 10, 1979.) 